\title{
Trajectory Control of Very Flexible Aircraft with Gust Disturbance
}

\author{
Matthew J. Dillsaver* Carlos E.S. Cesnik ${ }^{\dagger}$ Ilya V. Kolmanovsky \\ University of Michigan, Ann Arbor, MI, 48109
}

\begin{abstract}
This paper considers control of a very flexible aircraft in the presence of gust disturbances. Longitudinal trajectory tracking is performed using a hierarchical control scheme with either a PID or a sliding mode outer loop controller and a dynamic inversion inner loop controller. A Linear Quadratic Gaussian controller based on a reduced order linear model of the system is used to track lateral commands. The closed loop responses are demonstrated in fully nonlinear simulations with gust disturbances. Finally, we demonstrate a coordinated control of the ailerons to reduce wing curvature excursions from trim values.
\end{abstract}

\section{Introduction}

The pursuit of Unmanned Air Vehicles with loiter times in days, weeks or even years is driving research into High Altitude, Long Endurance (HALE) aircraft. This class of aircraft has obvious benefits for military designers for use as an Intelligence, Surveillance and Reconnaissance platform or for communication relay, ${ }^{1}$ but is also being researched for civilian uses such as environmental sensing and cellular telephone relay. ${ }^{2}$ Aircraft optimized for HALE flight tend to be built with high-aspect ratio wings that are long and slender. This leads to large lift-to-drag ratio and maximizing aerodynamic efficiency. Additionally, designers attempt to minimize power consumption, causing the aircraft structural weight to be lowered to the maximum extent possible. This low structural weight means that HALE aircraft are also very flexible, with large wing deformations possible in flight.

The flexibility of HALE aircraft presents several challenges for control design. The first challenge is the reduced separation, and possible overlap, between the lowest frequency elastic modes and the flight dynamic frequencies. In conventional aircraft control, a notch-filter may be used to avoid exciting the elastic modes, however this is not feasible in VFA due to the frequency overlap. Other challenges include a time lag in aircraft response to control inputs ${ }^{3}$ caused by the wing flexibility, and non-minimum phase zeros in the linearized equations of motion. Finally, very flexible aircraft (VFA) exhibit both structural and aerodynamic nonlinearities with the mass and stiffness matrices being state-dependent.

The same characteristics that allow VFA to be optimized for HALE flight can also cause them to be susceptible to external disturbances, such as wind gusts. This became apparent in 2003 when the AeroVironment Helios aircraft crashed into the Pacific Ocean near Hawaii. The accident investigation ${ }^{4}$ concluded that the aircraft encountered unexpected turbulence shortly after takeoff, causing it to enter into a high dihedral flight condition. This caused the aircraft to begin a divergent pitch oscillation with airspeed excursions doubling with every oscillation. The aircraft eventually broke apart after the design airspeed was exceeded. As a result, one of the recommendations from the NASA report was to develop more advanced, multi-disciplinary (structures, aeroelastic, aerodynamics, atmospheric, materials, propulsion, controls, etc.) time-domain analysis methods appropriate to highly-flexible morphing vehicles. ${ }^{4}$ Figure 1 shows the Helios aircraft in flight and compares it to XHALE, a smaller VFA developed at the University of Michigan.

There has been a growing amount of research into the dynamics of VFA. Patil, Hodges and Cesnik ${ }^{1}$ showed that there can be significant changes in aircraft dynamics as a result of large deformations seen in highly flexible wings. They also demonstrated that large changes are possible in VFA wing shape at differing

*Major, US Air Force, PhD Candidate (dills@umich.edu), Dept of Aerospace Engineering, Member, AIAA.

†Professor (cesnik@umich.edu), Dept of Aerospace Engineering, Fellow, AIAA.

¥Professor (ilya@umich.edu), Dept of Aerospace Engineering, Member, AIAA. 

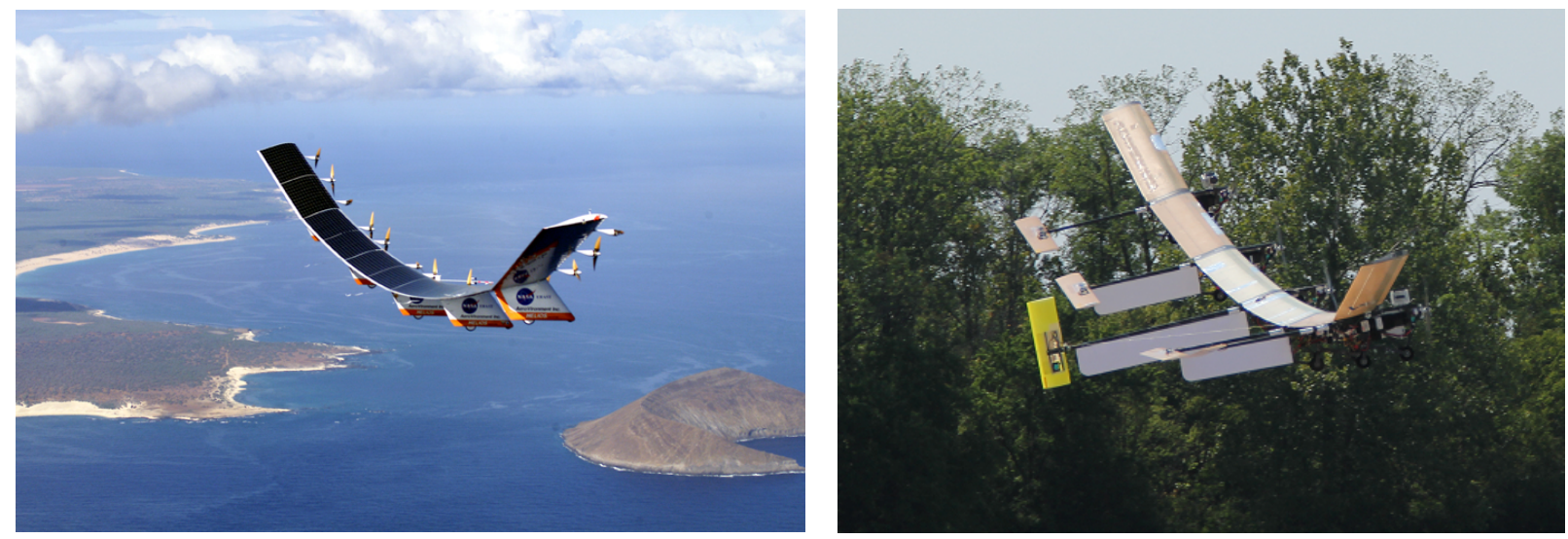

Figure 1. Helios ${ }^{4}$ (left) and XHALE (right) in flight.

flight conditions. Su and Cesnik ${ }^{5}$ compared the aerodynamic analysis of VFA using both the undeformed and deformed wing shapes and proved that this analysis must be done on the actual trimmed shape of the aircraft for a given flight condition. Zhou and $\operatorname{Ren}^{6}$ modeled the coupled dynamic behavior of a maneuvering flexible aircraft as a feedback-controlled multi-body system, leading to a time-domain method which can be used in aircraft conceptual design.

This paper considers control of a very flexible aircraft in the presence of a gust disturbance. A control approach based on Ref. 3 is implemented to track altitude change commands on a VFA model. An outer loop sliding mode controller is considered as an alternative to the PID controller of Ref. 3 to improve performance, and is combined with a dynamic inversion inner loop controller. Then, a new method for bank angle tracking using an LQG controller designed using a reduced order linear representation of the VFA model is demonstrated. After that, the longitudinal and lateral controllers are combined to demonstrate simultaneous tracking. The tracking performance is evaluated as the aircraft is subjected to various time-varying gust disturbances. Finally, we demonstrate a novel wing curvature reduction approach by coordinating ailerons using an LQG controller while tracking altitude commands in the presence of the gust disturbance.

\section{Background}

\section{A. VFA Modeling}

The aircraft model is designed and simulated using the University of Michigan Nonlinear Aeroelastic Simulation and Toolbox (UM/NAST). UM/NAST utilizes a strain based formulation of the elastic equations of motion, combined with rigid body equations and is capable of simulating rigid body, linearized and nonlinear aircraft dynamics. ${ }^{5}$ UM/NAST also uses an unsteady aerodynamics formulation based on the finite state inflow method of Peters and Johnson. ${ }^{7}$

The strain based formulation of the aeroelastic equations of motion (EOM) used in this work for flexible aircraft is derived using the principle of virtual work: ${ }^{8}$

$$
\begin{aligned}
M_{F F} \ddot{\varepsilon} & =-M_{F B} \dot{\beta}-C_{F F} \dot{\varepsilon}-C_{F B} \beta-K_{F F} \varepsilon+R_{F}, \\
M_{B B} \dot{\beta} & =-M_{B F} \ddot{\varepsilon}-C_{B B} \beta-C_{B F} \dot{\varepsilon}+R_{B}, \\
\zeta & =-\frac{1}{2} \Omega_{\zeta} \zeta, \\
\dot{p}_{b} & =\left[\begin{array}{ll}
C^{B G} & 0
\end{array}\right] \beta, \\
\dot{\lambda} & =F_{1} \ddot{q}+F_{2} \dot{q}+F_{3} \lambda,
\end{aligned}
$$

where $M$ is the generalized mass matrix, $C$ is the structural damping matrix, $K$ is the stiffness matrix, $R$ represents the generalized forces, the subscript ${ }_{F}$ denotes flexible terms, the subscript ${ }_{B}$ denotes terms in the body-fixed coordinate system, $\varepsilon$ is the strain vector containing the four strain elements (extensional 
strain, twist of the beam reference line, and bending about the $y$ and $z$ axes), $\beta$ is the rigid body velocity vector containing three translational and three rotational velocities, $\zeta$ represents the four quaternions, $p_{b}$ is a position vector for the body, $C^{B G}$ is a translation matrix for the body fixed coordinate system to the inertial system. The last equation calculates the unsteady aerodynamic forces and moments.

The coordinate system used in this formulation is based on a beam coordinate system and is shown in Figure 2. The global, G, frame is an inertial system fixed to the ground. Aircraft rigid body velocities are calculated in a body, B, frame which is centered at the origin which is an arbitrarily defined point and may not, in general, be located at the center of mass. In this system the positive $\mathrm{x}$-axis is out the right wing, the $\mathrm{y}$ axis is positive in the direction of forward flight, and the positive z-axis is up.

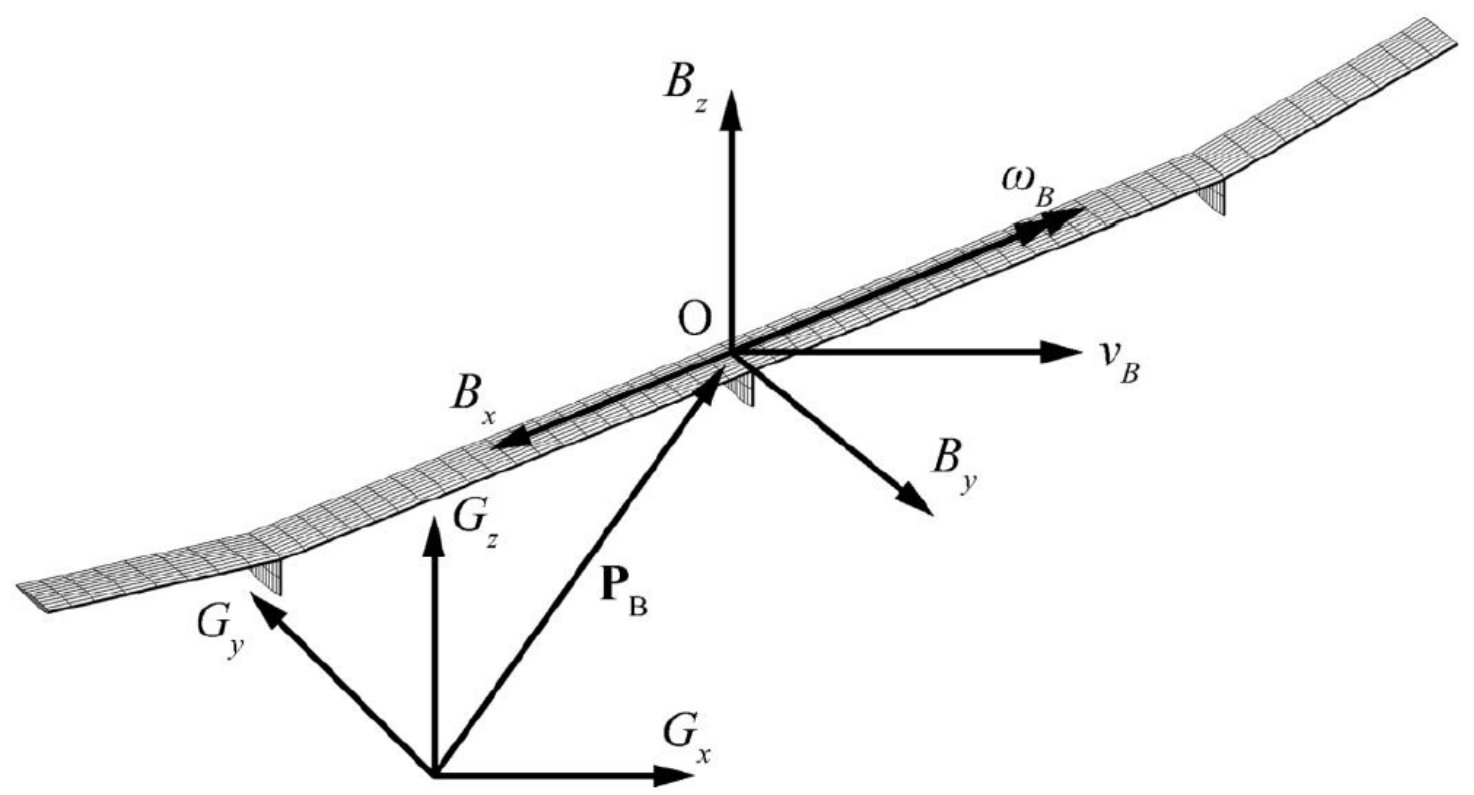

Figure 2. Coordinate systems used.

The VFA model used in this study is the XHALE, which is an aeroelastically representative test bed developed at the University of Michigan, similar to the aircraft in Ref 9 and show in Fig. 1. The aircraft has a $6 \mathrm{~m}$ wing span, with the outboard $1 \mathrm{~m}$ on each side having a dihedral angle of $10^{\circ}$. The wing has a rectangular cross-section, with a constant chord of $0.2 \mathrm{~m}$. The aircraft also has five motors, four horizontal, all moveable tails, as well as a center tail which can be either horizontal or vertical. The center tail is in the vertical position in this study. A UM/NAST representation of the aircraft is given in Fig. 3. The motors are not shown in the figure, but are installed on the five pods.

The longitudinal motion of the aircraft is controlled using the three center motors, acting together as a single control, and the four horizontal tails, acting together. The roll motion is controlled using the ailerons, which are controlled independently of each other, while the yaw is controlled using differential thrust on the two outboard motors, controlled independently. The aircraft is trimmed for straight and level, 1-g flight at $14 \mathrm{~m} / \mathrm{s}$ at an altitude of $30 \mathrm{~m}$. At this trim condition the wing tip is deflected up $0.38 \mathrm{~m}$, which is approximately $13 \%$ of the half-span.

The aircraft flight dynamic modes are shown in Table 1. Note that all modes are stable and relatively well damped, with the exception of the spiral mode, which is close to the stability boundary. Another important parameter when dealing with VFA is the separation between the lowest frequency elastic modes and the flight dynamic modes. For this aircraft, the first bending frequency occurs at $0.59 \mathrm{~Hz}$, thus there is only a frequency separation of $0.46 \mathrm{~Hz}$ between the phugoid and first bending modes.

\section{B. Gust Modeling}

There are many approaches to modeling wind gusts for aircraft design and simulations. The frequently used formulations are based on either stochastic or discrete methods. The discrete gust method forms a gust 


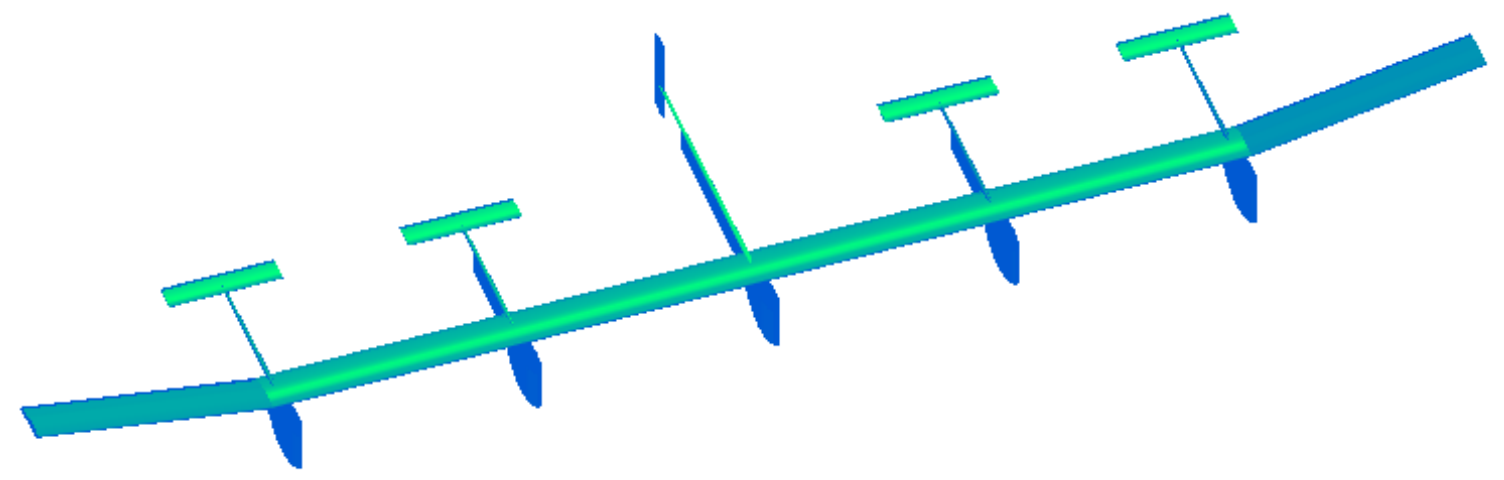

Figure 3. UM/NAST depiction of VFA model.

Table 1. VFA model flight dynamic modes.

\begin{tabular}{lllll}
\hline \hline Phugoid & Short Period & Dutch Roll & Roll & SPIRAL \\
\hline$-0.209 \pm 0.836 i$ & $-8.3204 \pm 12.097 i$ & $-1.098 \pm 1.427 i$ & -7.49 & -0.0015 \\
\hline \hline
\end{tabular}

field with a one-minus-cosine distribution. For example, the Federal Aviation Regulations (FAR) Part $23^{10}$ prescribe a discrete model given by:

$$
U=\frac{U_{d e}}{2}\left[1-\cos \left(\frac{2 \pi s}{25 C}\right)\right],
$$

where $U$ is the gust velocity, $s$ is the distance penetrated into the gust, $C$ is the mean geometric chord and $U_{d e}$ is the derived gust velocity which varies from 33 to $66 \mathrm{ft} / \mathrm{s}$ depending on the altitude, aircraft category and air roughness. ${ }^{10}$ The Defense Advanced Research Project Agency (DARPA) defined a discrete, non-uniform spanwise gust model for use in the design of the Vulture II program. ${ }^{11}$ This model uses a one-minus-cosine temporal distribution with a sinusoidal spanwise variation, considering the gust as a vertical disturbance only. The single-amplitude gust velocity is given by:

$$
U_{S A}=\frac{U_{d e}}{2}\left(\frac{b}{2 L}\right)^{\frac{1}{3}},
$$

where $L$ is the turbulence scale and $b$ is the aircraft wing span. The gust formulation used in this study was developed in Ref. 12 and is based on the DARPA model given by Eq. 7. The gust velocity amplitude at the aircraft center is determined by:

$$
U_{d e}=\frac{U_{r e f}}{2}\left[1-\cos \left(2 \pi \frac{t}{t_{g}}\right)\right],
$$

where $U_{r e f}$ is the reference gust velocity, $t$ is the current time and $t_{g}$ is the gust duration. The gust velocity calculated using Eqs. 7 and 8 is applied at 111 points along the aircraft wing in the UM/NAST model. In this study, the gust velocity is assumed to be constant along the span of the aircraft.

In the stochastic formulations, such as the Dryden and von Karman models, the gust is considered a stationary, random and Gaussian process. Then properties of the turbulence field such as the turbulence length scale and RMS gust velocity are used to determine the power spectral density (PSD) of the gust. ${ }^{13}$ The PSD for the Dryden model is given by: ${ }^{13}$

$$
\Phi_{d}(\Omega)=\sigma^{2} \frac{L}{\pi}\left(\frac{1+3 L^{2} \Omega^{2}}{\left(1+L^{2} \Omega^{2}\right)^{2}}\right),
$$


where $\sigma$ is the root mean square gust velocity, $L$ is the turbulence length, and $\Omega$ is the spatial frequency which is defined as

$$
\Omega=\frac{\omega}{v_{y}},
$$

where $\omega$ is the angular frequency of the gust and $v_{y}$ is the forward velocity of the aircraft.

In practice, a time history of the gust is created by passing a white noise signal through a coloring filter. The coloring filter, $G(s)$ is designed so that the output signal $\Phi(\omega)$ will have the desired PSD. An exact coloring filter for the Dryden spectrum can be determined while only an approximate filter can be determined for the von Karman model. However, flight test data have shown a better agreement with the von Karman PSD than with the Dryden PSD. ${ }^{13}$ This leads to a tradeoff that one wishing to simulate gust response must make. The coloring filter for the Dryden profile is given by: ${ }^{13}$

$$
G_{d r y}(s)=\sigma_{w} \sqrt{\frac{\tau}{\pi}} \frac{1+\sqrt{3} \tau s}{(1+\tau s)^{2}},
$$

where $\tau=L / v_{y}$. For the approximate filter in the case of the von Karman model, see Ref. 13. Our subsequent stochastic gust simulations are based on the Dryden model, and the gust profile is passed through a low pass filter to smooth the signal. While these gust models were developed for large aircraft flying at high altitudes, they are also applicable to smaller aircraft because the gust is a function of aircraft span, and different values of the turbulence scale, $L$, can be used for low altitude flight.

\section{Control Architecture}

The control architecture chosen for this study is based on a two-loop scheme developed by Shearer and Cesnik $^{3}$ to control VFA, with a block diagram for longitudinal control shown in Figure 4. This scheme was designed based on a heuristic approach mimicking a human pilot. It consists of a stable inner loop in which the states are the linear and angular velocities, $\beta$, augmented with appropriate error states. The outer loop consists of a nonlinear transformation as well as an outer loop controller. The inner loop controller was tuned first, and after adequate performance was obtained tracking $\beta$ values, the outer loop was tuned. The following assumptions are made, following Ref 3:

1) The angle of sideslip is zero;

2) The Euler roll angle $\phi$ is used in place of the bank angle $\mu$;

3) Total velocity $V_{t}$ is prescribed and constant;

4) The angle of attack, $\alpha$ is proportional to $\cos \phi$.

The outer loop controllers considered in this study are a PID controller, as well as a sliding mode controller. The various inner loop controllers used are designed using dynamic inversion, Linear Quadratic Regulator (LQR), and a Linear Quadratic Gaussian (LQG) control applied to a reduced order linear model of the aircraft. The theoretical background for these control techniques is presented in the appendix.

\section{XHALE Modeling, Controller Design, and Numerical Studies}

\section{A. Longitudinal Control}

The design steps to implement the control approach in Fig. 4 for the XHALE aircraft are now detailed. The inner loop controller for the longitudinal motion is based on a dynamic inversion scheme developed by Shearer and Cesnik. ${ }^{3}$ See the Appendix for the background on dynamic inversion. To develop an inner loop dynamic inversion controller, consider the model (1) - (2), re-written in matrix form as

$$
\left[\begin{array}{ll}
M_{F F} & M_{F B} \\
M_{B F} & M_{B B}
\end{array}\right]\left[\begin{array}{c}
\ddot{\varepsilon} \\
\dot{\beta}
\end{array}\right]=-\left[\begin{array}{ll}
C_{F F} & C_{F B} \\
C_{B F} & C_{B B}
\end{array}\right]\left[\begin{array}{c}
\dot{\varepsilon} \\
\beta
\end{array}\right]-\left[\begin{array}{c}
K_{F F} \varepsilon \\
0
\end{array}\right]+\left[\begin{array}{c}
R_{F} \\
R_{B}
\end{array}\right] .
$$

From (12) it follows that $\ddot{\varepsilon}$ and $\dot{\beta}$ are given by

$$
\ddot{\varepsilon}=-C_{11} \dot{\varepsilon}-C_{12} \beta-Q_{M} K_{F F} \varepsilon+R_{F 1},
$$




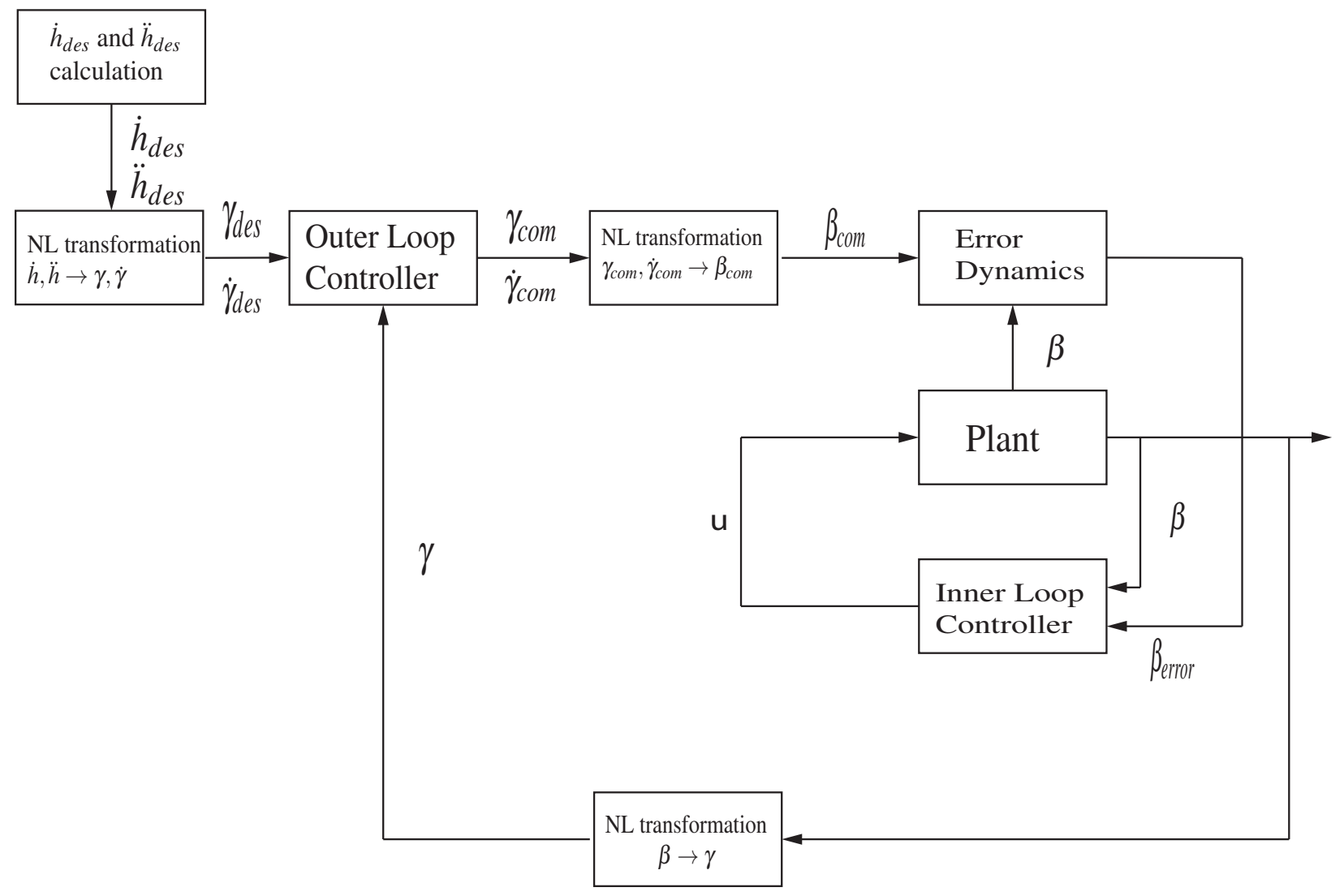

Figure 4. Longitudinal control architecture based on Ref. 3. 


$$
\dot{\beta}=-C_{22} \beta-C_{21} \dot{\varepsilon}+R_{B 1},
$$

where

$$
\begin{aligned}
Q_{M} & =\left(M_{F F}-M_{F B} M_{B B}^{-1} M_{B F}\right)^{-1}, \\
C_{11} & =Q_{M} C_{F F}-Q_{M} M_{F B} M_{B B}^{-1} C_{B F}, \\
C_{12} & =Q_{M} C_{F B}-Q_{M} M_{F B} M_{B B}^{-1} C_{B B}, \\
C_{21} & =-M_{B B}^{-1} M_{B F} Q_{M} C_{F F}+\left(M_{B B}^{-1} M_{B F} Q_{M} M_{F B} M_{B B}^{-1}+M_{B B}^{-1}\right) C_{B F}, \\
C_{21} & =-M_{B B}^{-1} M_{B F} Q_{M} C_{F B}+\left(M_{B B}^{-1} M_{B F} Q_{M} M_{F B} M_{B B}^{-1}+M_{B B}^{-1}\right) C_{B B}, \\
R_{F 1} & =Q_{M} R_{F}-Q_{M} M_{F B} M_{B B}^{-1} R_{B}, \\
R_{B 1} & =-M_{B B}^{-1} M_{B F} Q_{M} R_{F}+\left(M_{B B}^{-1} M_{B F} Q_{M} M_{F B} M_{B B}^{-1}+M_{B B}^{-1}\right) R_{B} .
\end{aligned}
$$

Then the generalized force vectors $R_{F 1}$ and $R_{B 1}$ can be re-written in control affine form leading to a new form for Eq. 14

$$
\dot{\beta}=f_{\beta}+g_{\beta} u_{\text {long }},
$$

where $u_{\text {long }}$ is the control vector consisting of commands to the elevators and center three motors. The dynamic inversion controller is now derived based on Eq. 22. The outputs to be tracked using this controller are the forward velocity $v_{B_{y}}$ and the pitch rate, $\omega_{B_{x}}$ that form the output vector, $y_{\text {long }}$ :

$$
y_{\text {long }}=C_{\text {long }} \beta \text {, }
$$

where

$$
C_{\text {long }}=\left[\begin{array}{llllll}
0 & 1 & 0 & 0 & 0 & 0 \\
0 & 0 & 0 & 1 & 0 & 0
\end{array}\right] .
$$

The control vector for the two longitudinal inputs, elevator deflection and thrust, is prescribed in terms of the pseudo-control $v_{\text {long }}$ as

$$
u_{\text {long }}=g_{\beta}^{-1}\left(v_{\text {long }}-f_{\beta}\right),
$$

leading to $\dot{\beta}=v_{\text {long }}$. The pseudo-control $v_{\text {long }}$ is found by applying LQR techniques to the linear system model augmented with the integral states

$$
\left[\begin{array}{c}
\dot{\beta} \\
\dot{z}_{\text {long }}
\end{array}\right]=\left[\begin{array}{cc}
0 & 0 \\
C_{\text {long }} & 0
\end{array}\right]\left[\begin{array}{c}
\beta \\
z_{\text {long }}
\end{array}\right]+\left[\begin{array}{c}
I \\
0
\end{array}\right] v_{\text {long }}-\left[\begin{array}{c}
0 \\
I
\end{array}\right]\left[\begin{array}{c}
v_{B y, c o m} \\
v_{B z, c o m} \\
\omega_{B x, c o m}
\end{array}\right],
$$

where $v_{B y, c o m}, v_{B z, \text { com }}$ and $\omega_{B x, \text { com }}$ denote, respectively, the commanded forward velocity, vertical velocity and pitch rate, and $z$ denotes the integral state vector. Additionally, $f_{\beta}$ and $g_{\beta}$ in Eq. 25 are calculated at every time step based on the current aircraft states.

The outer loop is designed to act similarly to a human pilot. Referring to Fig. 4, suppose the outer loop is given an altitude change command. The aircraft is then commanded to climb at a constant climb rate, $\dot{h}$ until $90 \%$ of the climb has been completed, and the desired flight path angle $\gamma$ necessary to achieve this $\dot{h}$ is calculated. After $90 \%$ of the climb has been completed, the controller switches to computing the $\dot{\gamma}$ and $\gamma$ required to reduce the altitude error to zero in a given amount of time. These desired values of $\dot{\gamma}$ and $\gamma$, as well as the current values, are then passed through an outer loop controller, which calculates the commanded values of $\dot{\gamma}$ and $\gamma$, denoted by $\gamma_{c o m}$ and $\dot{\gamma}_{c o m}$ in Fig. 4 . These commanded values are then passed though a nonlinear transformation which converts them to commanded $\beta$ values, $\beta_{\text {com }}$, to be passed to the inner loop. 
The nonlinear transformation converts the flight path angle $\gamma$ and roll angle $\phi$ as well as their time derivatives $\dot{\gamma}$ and roll angle $\dot{\phi}$ to body velocities. It begins by calculating the current angle of attack and its time derivative: ${ }^{3}$

$$
\begin{aligned}
\alpha & =\frac{1}{\cos \phi} \alpha_{0}, \\
\dot{\alpha} & =\tan \phi \sec \phi \alpha_{0} \dot{\phi},
\end{aligned}
$$

where $\alpha_{0}$ is the trimmed, wings-level angle of attack. Next the body translational velocities, with $v_{x}=0$ by the assumptions above, are found using ${ }^{3}$

$$
\begin{aligned}
& v_{z}=-V_{t} \sin \alpha, \\
& v_{y}=\sqrt{V_{t}^{2}-v_{z}^{2}},
\end{aligned}
$$

where $V_{t}=\sqrt{v_{x}^{2}+v_{y}^{2}+v_{z}^{2}}$ is the total velocity. Next, the Euler pitch angle and its time derivative are calculated by ${ }^{3}$

$$
\begin{aligned}
\sin \gamma & =\cos \alpha \sin \theta-\cos \phi \sin \alpha \cos \theta \\
\dot{\theta} & =\frac{\cos \gamma \dot{\gamma}+(\sin \alpha \sin \theta+\cos \phi \cos \alpha \cos \theta) \dot{\alpha}}{\cos \alpha \cos \theta+\cos \phi \sin \alpha \sin \theta}-\frac{\sin \phi \sin \alpha \cos \theta \dot{\phi}}{\cos \alpha \cos \theta+\cos \phi \sin \alpha \sin \theta} .
\end{aligned}
$$

Then the body angular velocities are found by simultaneously solving the following system of equations ${ }^{3}$ for $\omega_{x}, \omega_{y}$, and $\omega_{z}$

$$
\begin{aligned}
\dot{\phi} & =(\tan \theta \sin \phi) \omega_{x}+\omega_{y}-(\tan \theta \cos \phi) \omega_{z}, \\
g \sin \phi \cos \phi & =v_{z} \omega_{y}-v_{y} \omega_{z}, \\
\dot{\theta} & =\cos \phi \omega_{x}+\sin \phi \omega_{z},
\end{aligned}
$$

where $g$ is the gravity.

The nonlinear transformations employed in the design of the inner loop controller and command transformations entail errors necessitating the use of an outer loop controller to correct them. In Ref. 3, the following outer loop control structure is employed

$$
\begin{aligned}
& \gamma_{c o m}=K_{\gamma} \Delta \gamma+K_{I \gamma} \int_{0}^{t} \Delta \gamma d \tau+K_{I I \gamma} \int_{0}^{t}\left(\int_{0}^{\tau} \Delta \gamma d s\right) d \tau+K_{d \gamma} \Delta \dot{\gamma}, \\
& \dot{\gamma}_{c o m}=K_{\dot{\gamma}} \Delta \dot{\gamma}+K_{I \dot{\gamma}} \Delta \gamma+K_{I I \dot{\gamma}} \int_{0}^{t} \Delta \gamma d \tau+K_{I I I \dot{\gamma}} \int_{0}^{t}\left(\int_{0}^{\tau} \Delta \gamma d s\right) d \tau
\end{aligned}
$$

where $\Delta \gamma=\gamma-\gamma_{\text {des }}$ and $\Delta \dot{\gamma}=\dot{\gamma}-\dot{\gamma}_{\text {des }}$. we refer to (36) - (37) as a (non-standard) PID controller.

Several additional design steps of the outer loop controller (versus Ref. 3) have been undertaken in this work for XHALE aircraft. To motivate these, suppose the aircraft is given a climb of $20 \mathrm{~m}$, which corresponds to 100 chord lengths, with a desired climb rate of $0.432 \mathrm{~m} / \mathrm{s}$. This climb rate is the climb rate the Helios had on its mishap flight. ${ }^{4}$ The response with an outer loop PID controller is shown in Fig. 5. The aircraft follows well the climb command, but becomes unstable while trying to level off at $20 \mathrm{~m}$. When examining the plots of speed and pitch rate, they track the commands well, meaning the problem is with the outer loop. The figure also shows that $\gamma$ seems to be out of phase with $\gamma_{c o m}$.

Two approaches to increase the robustness of the outer loop controller have been pursued. The first approach is to increase the gains of the PID controller when the aircraft finishes $90 \%$ of the climb. We refer to this approach as the Higher Gain PID approach even though the gains are increased only for the level off phase. The second approach is to design a sliding mode controller to ensure convergence of $\gamma$ to $\gamma_{\text {des }}$. For the second approach, we approximate the plant dynamics as a double integrator of the form

$$
\ddot{\gamma}=u,
$$



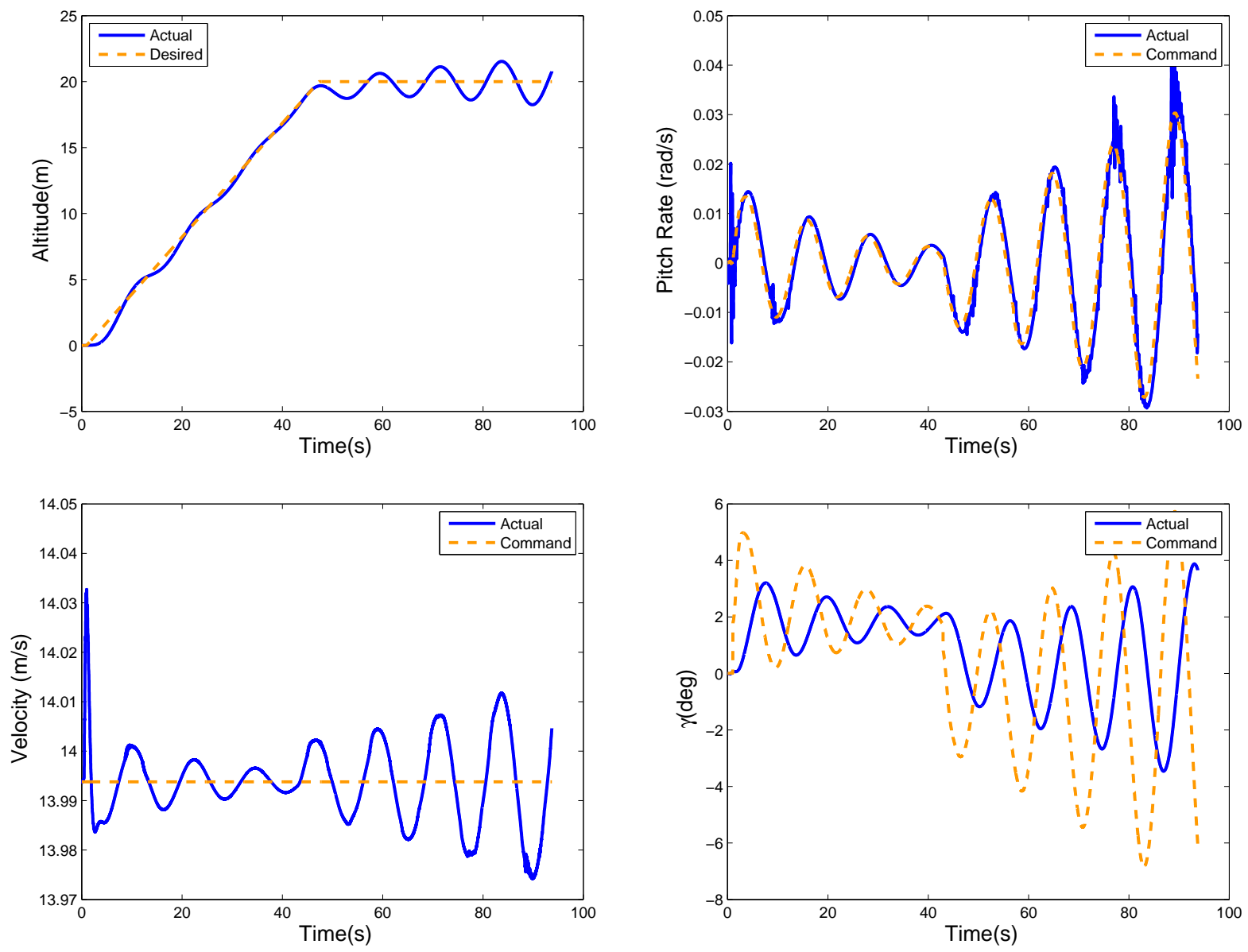

Figure 5. Response to altitude change command. 
where $u$ is $\dot{\gamma}_{c o m}$ in Fig. 4 and $\gamma_{c o m}$ is set to $\gamma_{d e s}$. We rely on sliding mode control robustness to compensate for unmodeled dynamics.

The goal of sliding mode control is to force the trajectories of a system to a user-defined sliding surface. The sliding surface is defined by $S=0$, where

$$
S=\lambda\left(\gamma-\gamma_{\text {des }}\right)+\left(\dot{\gamma}-\dot{\gamma}_{\text {des }}\right) .
$$

Choose a candidate Lyapunov function of the form

$$
V(x)=\frac{1}{2} S^{2},
$$

then from Eqs. 38 - 40 it follows that

$$
\begin{aligned}
\dot{V}(x) & =S \dot{S} \\
& =S\left[\lambda\left(\dot{\gamma}-\frac{d}{d t} \gamma_{\text {des }}\right)+\left(\ddot{\gamma}-\frac{d}{d t} \dot{\gamma}_{\text {des }}\right)\right] \quad=S\left[\lambda\left(\dot{\gamma}-\frac{d}{d t} \gamma_{\text {des }}\right)+u-\frac{d}{d t} \dot{\gamma}_{\text {des }}\right] .
\end{aligned}
$$

Selecting

$$
u=-\left[\lambda\left(\dot{\gamma}-\frac{d}{d t} \gamma_{d e s}\right)-\frac{d}{d t} \dot{\gamma}_{\text {des }}\right]-K \operatorname{sign}(S),
$$

with $K>0$ ensures that $\dot{V}=-K|S|<0$ and that $S$ converges to zero in finite time. Therefore $\gamma(t) \rightarrow \gamma_{\text {des }}(t)$ and $\dot{\gamma}(t) \rightarrow \dot{\gamma}_{\text {des }}(t)$ as $t \rightarrow \infty$.

One problem with implementing a sliding mode controller is that it is discontinuous and the closed loop system is prone to chattering. ${ }^{14}$ To address this, a boundary layer of width $\Phi$ is created around $S=0$ so that $u$ is given by ${ }^{14}$

$$
u=-\left[\lambda\left(\dot{\gamma}-\frac{d}{d t} \gamma_{d e s}\right)-\frac{d}{d t} \dot{\gamma}_{d e s}\right]-K s a t\left(\frac{S}{\Phi}\right),
$$

where $\operatorname{sat}(\cdot)$ is the saturation function.

\section{B. Numerical Case Studies for Longitudinal Control}

The responses of three outer loop controllers are shown in Fig. 6. Both the higher gain PID and sliding mode controllers perform well at matching the altitude command. Both controllers produce almost no overshoot and maintain the desired speed, however the sliding mode controller has larger excursions. The tracking is done with small control effort for both controllers. Note that, the original PID outer loop controller results in infeasible negative thrust commands.

Next, the aircraft is given the same altitude change command, but this time with a gust disturbance. The gusts used were $2 \mathrm{~s}, 4 \mathrm{~s}$, and $8 \mathrm{~s}$ duration, repeating 1-cos discrete gusts as well as a Dryden gust profile, with the gust acting only in the vertical direction. The reference gust amplitude in Eq. 8 was $5 \mathrm{~m} / \mathrm{s}$ and the Dryden gust amplitude was created using the filter in Eq 11 and was scaled so that the maximum gust amplitude was equal to that of the discrete gust. The output of the coloring filter for the Dryden gust was also passed through a low pass filter in order to smooth out the profile. The 8s repeating 1-cos gust as well as the filtered Dryden gust profile are shown in Fig. 7.

The responses of the aircraft to the climb command in the presence of the gusts are shown in Fig. 8. The figures show good tracking for both controllers with all four gusts. The higher gain PID controller responds slightly slower at the top of the climb, with a similarly small overshoot to the sliding mode controller.

The root curvature on the right side of the aircraft is shown in Fig. 9. The higher gain PID controller has larger root curvature values in the 2 s duration gust, but smaller is the other cases. Since root curvature is proportional to bending moment, this means that the aircraft with the higher gain PID controller would induce smaller bending moments than the sliding mode controller experiencing the same gust in these cases. 

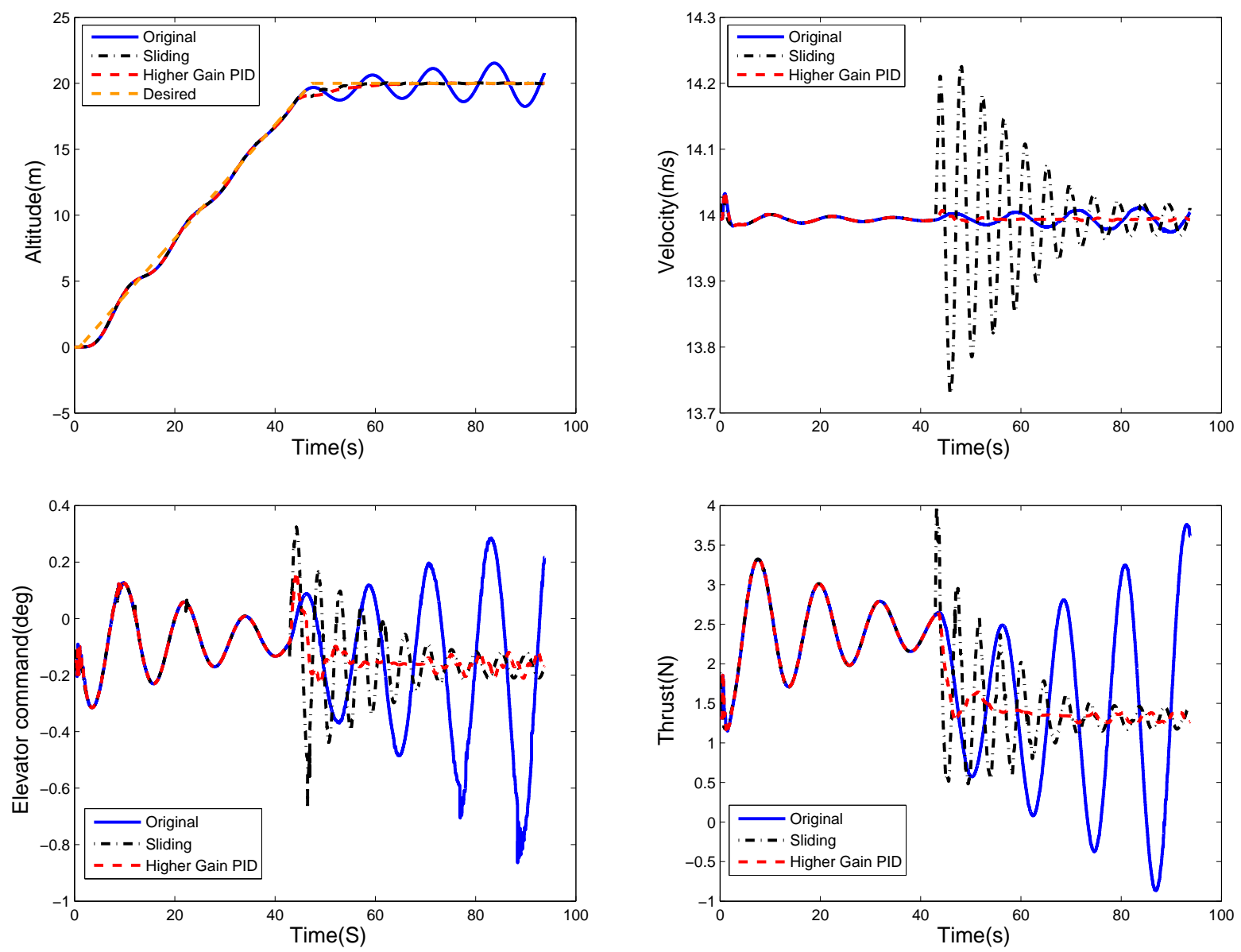

Figure 6. Comparison of outer loop controllers.
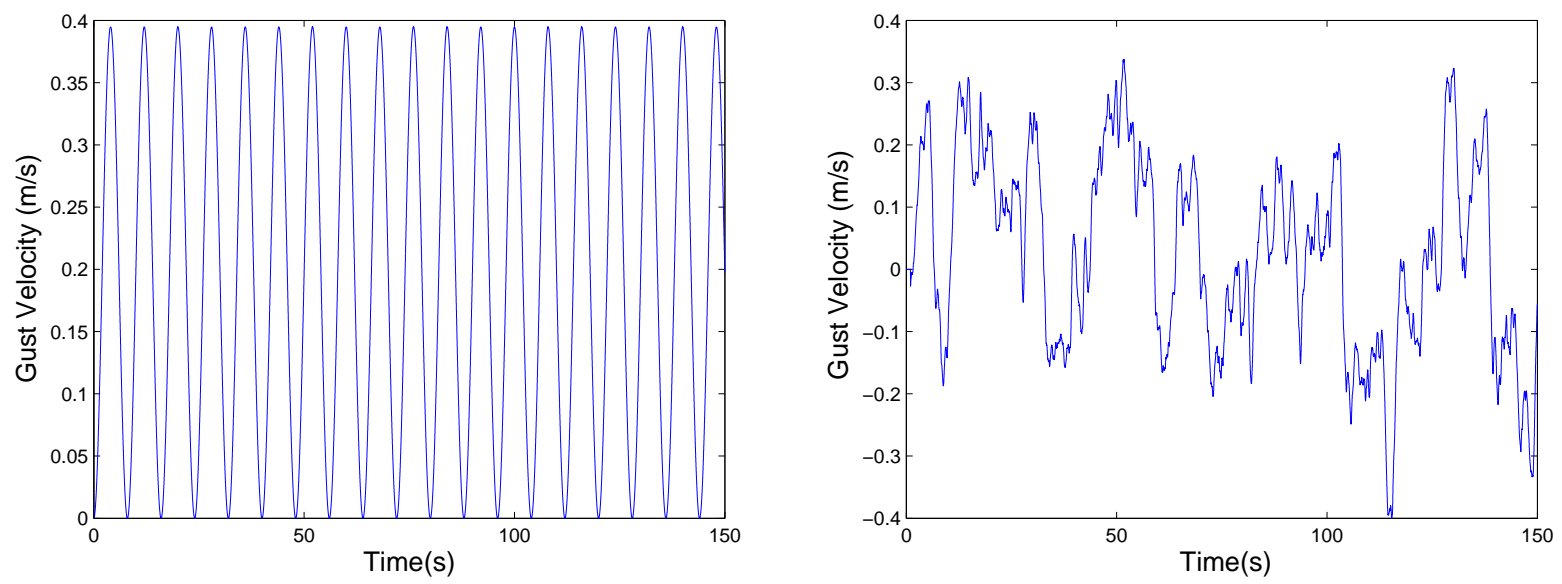

Figure 7. Gust profiles for 8 s duration, repeating discrete gust (left) and Dryden profile (right). 

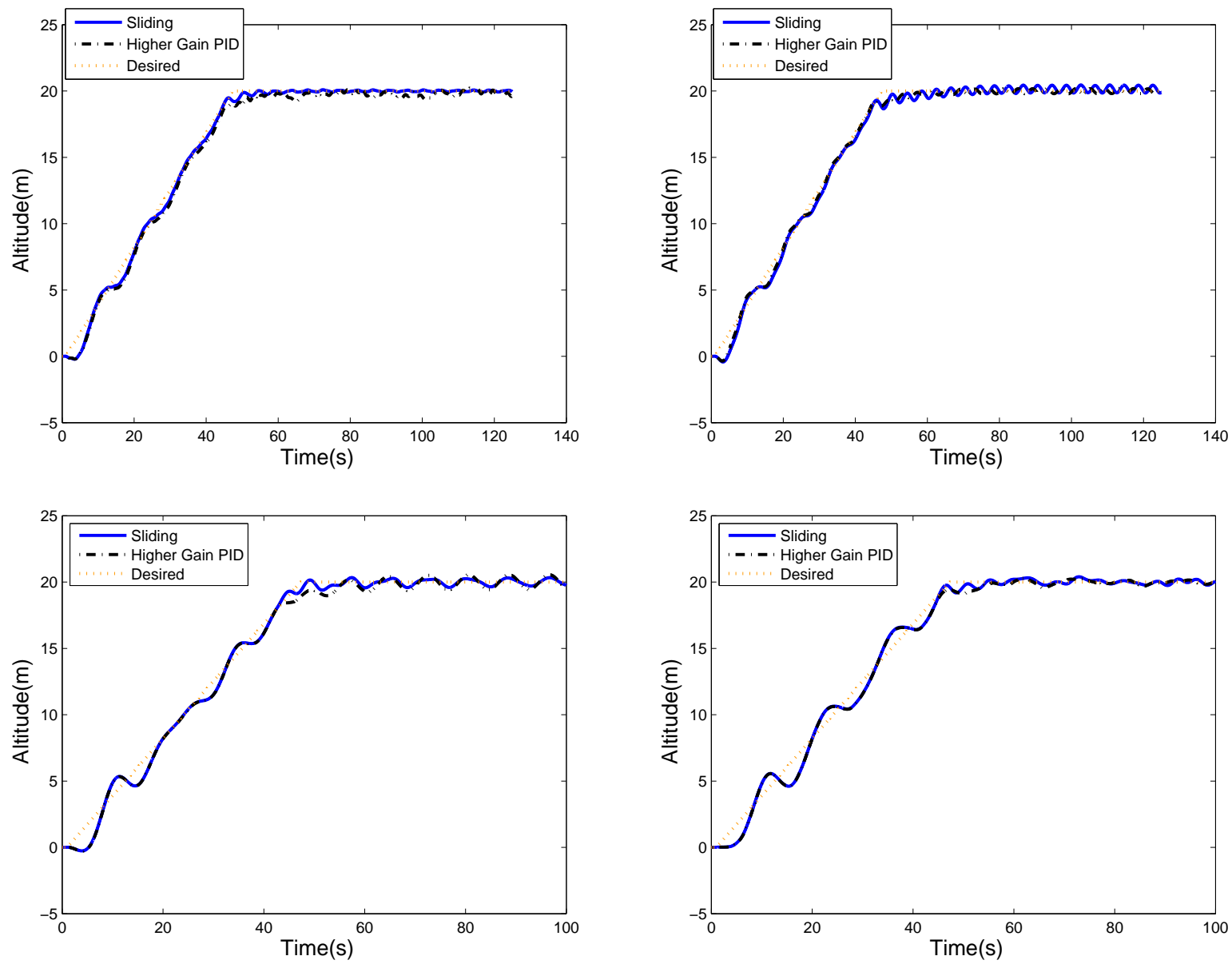

Figure 8. Altitude tracking response for $2 \mathrm{~s}$ (top left), 4s (top right), 8s (bottom left) and Dryden gust (bottom right). 

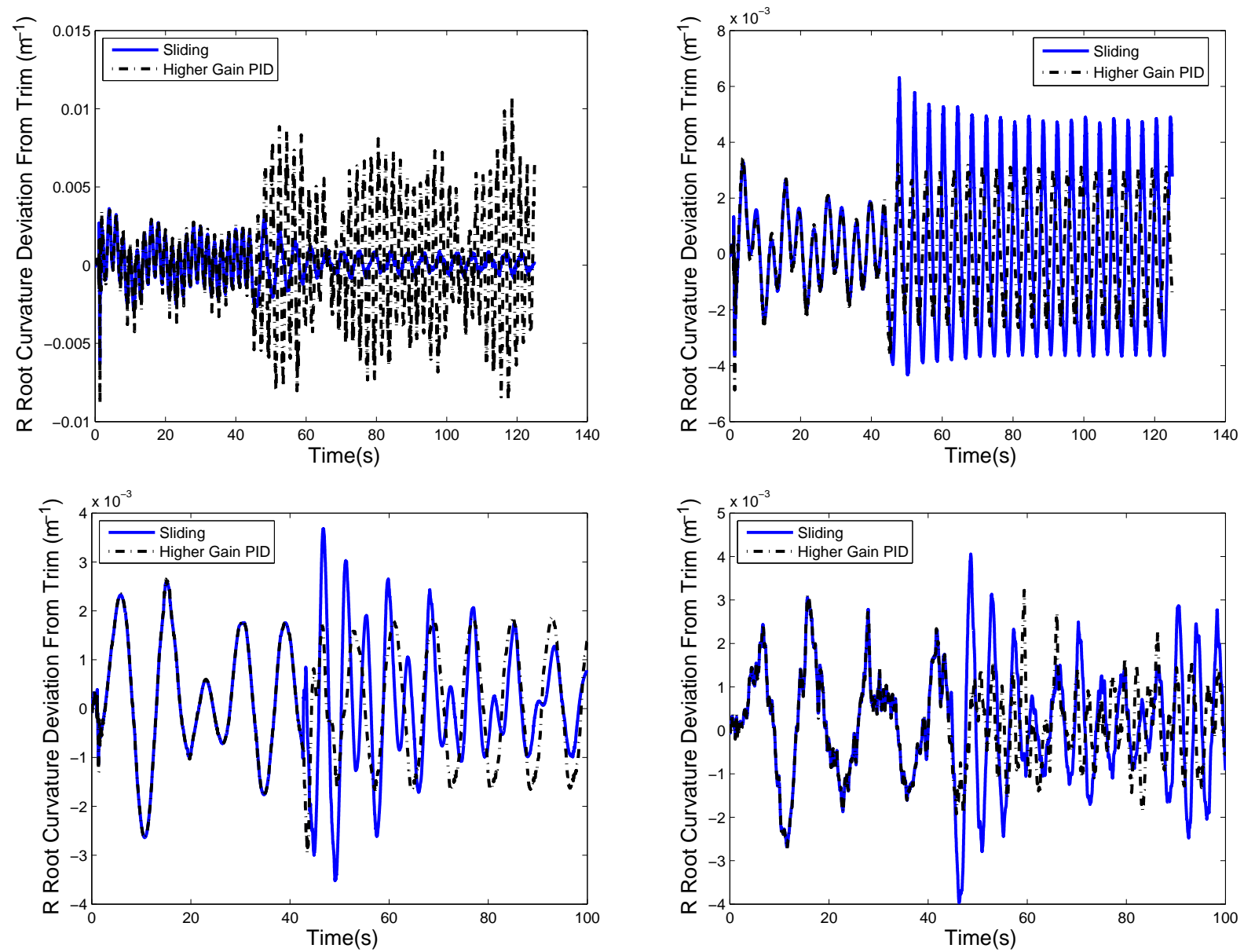

Figure 9. Root curvature response during altitude tracking response for $2 \mathrm{~s}$ (top left), $4 \mathrm{~s}$ (top right), $8 \mathrm{~s}$ (bottom left) and Dryden gust (bottom right). 


\section{Lateral Control}

The lateral controller is designed using a reduced order linear model of the aircraft and the LQG approach. The nonlinear equations are first linearized and then balanced truncation is used to create a $50^{t h}$ order model. The output vector, $y$ is:

$$
y=\left[\begin{array}{c}
\kappa_{y, \text { rroot }} \\
\kappa_{y, \text { lroot }} \\
v_{x} \\
\omega_{y} \\
\omega_{z} \\
\psi \\
\phi \\
\kappa_{y, \text { rmid }} \\
\kappa_{y, l m i d}
\end{array}\right]
$$

where $\kappa_{y}$ is the wing curvature, $r$ and $l$ denote right and left, respectively, root and mid are measured at the root and mid-span of the wing, $\psi$ and $\phi$ are the Euler yaw and roll angles. The choice to use four wing curvature values along the wing was made in order to allow the controller to get an accurate picture of the entire wing shape, which will not be symmetric during roll maneuvers. The four control inputs are the left and right ailerons, and the left and right outboard motors, with all four able to move independently.

An LQG is then designed using the output vector, $y$, and the reduced order linearization. The resulting state-space controller model is then discretized and added to the UM/NAST simulation.

\section{Numerical Case Studies for Lateral Control}

The aircraft is given a single period square wave roll command with an amplitude of $5^{\circ}$ and a yaw angle command of zero. In order to make a coordinated turn, there would be a need to follow a non-zero yaw command. In this case, a yaw angle of zero is somewhat arbitrarily chosen to demonstrate tracking of a prescribed value. The response is shown in Fig. 10. This control scheme has no guarantee of steady state error and the roll angle has a significant steady state error, but the error is approximately the same in both the positive and negative directions.
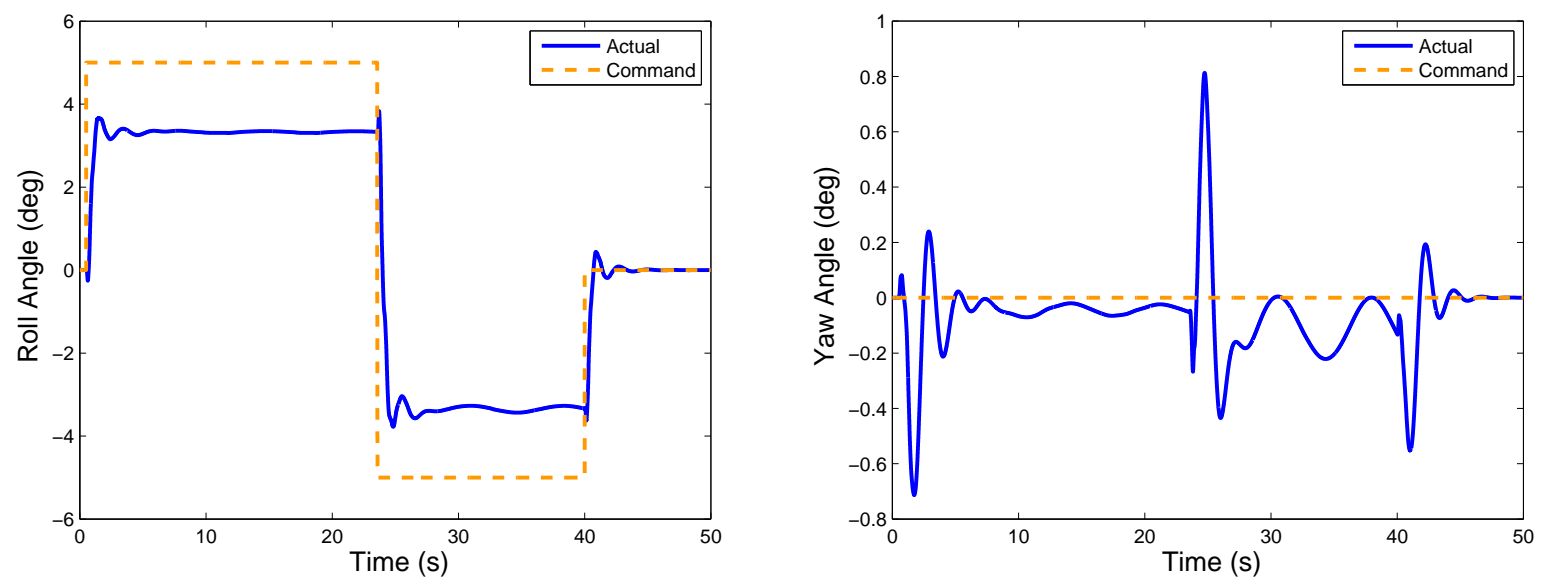

Figure 10. Roll tracking response.

In order to remove this steady state error, a constant pre-compensator was added to the roll command (alternatively, integral action on the tracking error could have been added). Additionally, the aircraft was also given a $10^{\circ}$ square wave roll command to verify the same pre-compensator would work at other command angles. The results are shown in Fig. 11. The responses are good and the aircraft can track the commands of both amplitudes. 

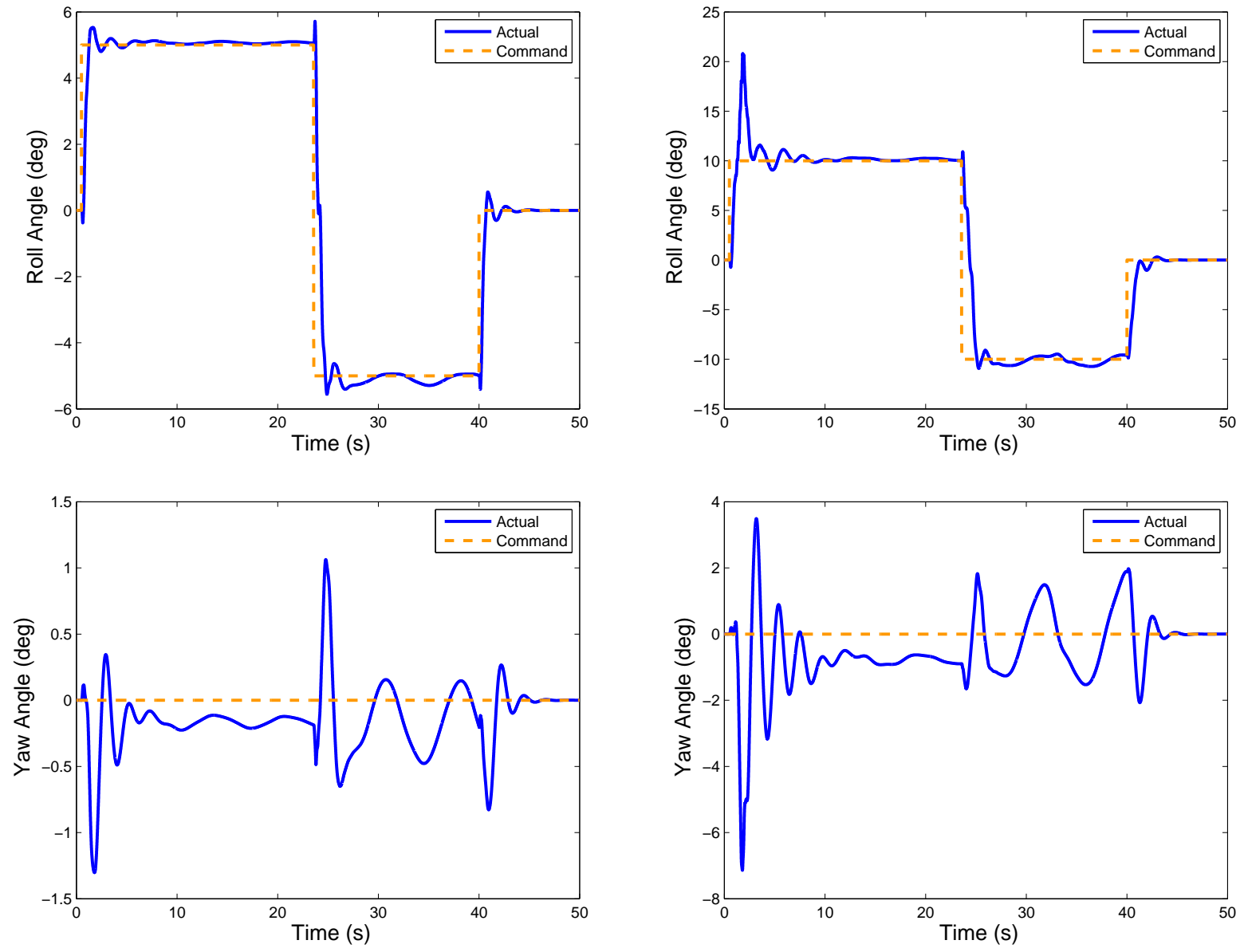

Figure 11. Roll tracking response with precomensator to commands of $5^{\circ}$ (left) and $10^{\circ}$ (right). 
The aircraft was then given the same roll tracking command in the presence of the 8s duration repeating gust disturbance with the response shown in Fig. 12. As the figure shows, there is almost no difference in the response between the aircraft in still air and in the gust.
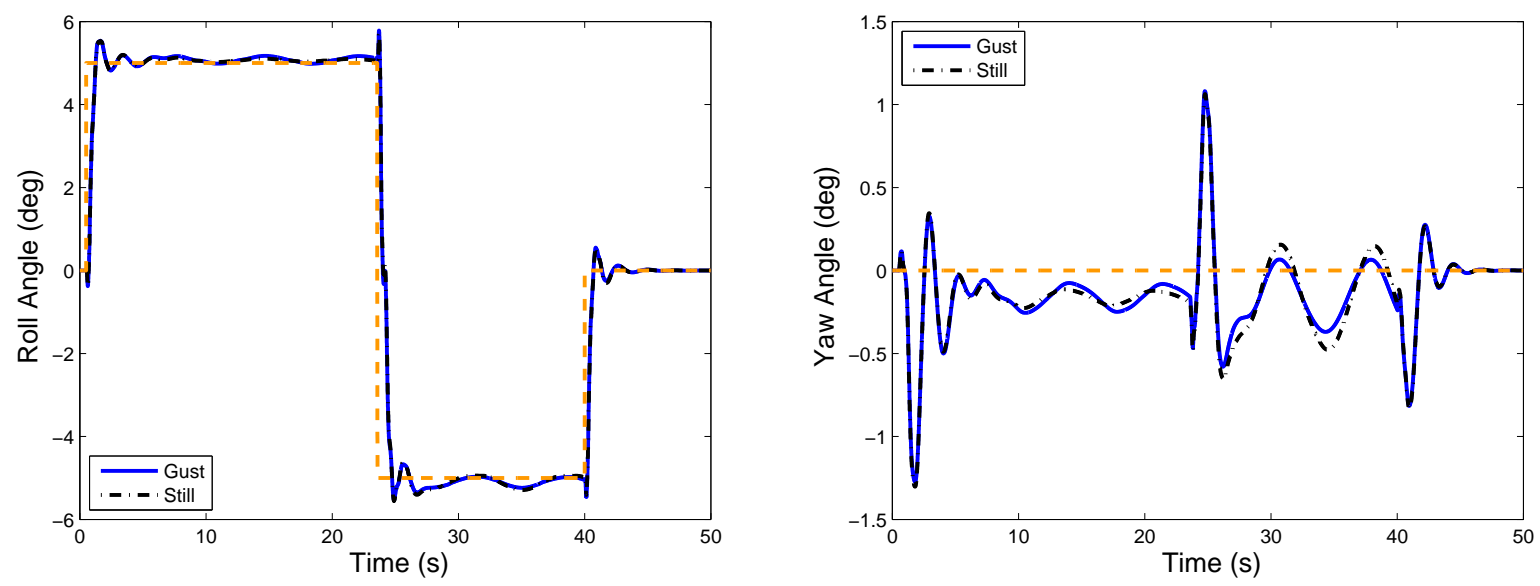

Figure 12. Roll tracking response with gust disturbance.

\section{E. Combined Trajectory Control}

The aircraft response to the simultaneous climb and roll commands described with both the higher gain PID and sliding mode controllers in still air are shown in Fig. 13. The aircraft is able to track all commands, with the altitude tracking degrading somewhat when compared with the climb tracking only plots. The sliding mode controller slightly overshoots the altitude, while the higher gain PID does not.

\section{F. Gust Load Reduction}

Finally, we describe an approach to coordinated control of ailerons to reduce wing curvatures induced by maneuvering flight and by the gusts. The controller is an LQR that uses the entire vector of elastic states as well as error states on the right and left root curvatures for feedback. Since the wing curvatures are proportional to bending moment, this approach minimizes the bending moments of the aircraft. All of the elastic states, as well as the error states, use the trim values as the baseline, meaning the goal of the controller is to have the aircraft remain at the trimmed shape while maneuvering with a gust disturbance.

The aircraft was first given the same $20 \mathrm{~m}$ climb command as above, but with the ailerons actively reducing the wing curvatures. The results are shown in Figs. $14-17$. The altitude tracking performance for the controller with the curvature reducing LQR is virtually identical to the original controller. The root curvature departure from trim values, and therefore the bending moment, caused by the gust and maneuvering, are significantly reduced in all the gusts, but the amount of the reduction is dependent on the gust durations. The $2 \mathrm{~s}$ duration gust simulation had the lowest reduction in curvature at $31 \%$, while the $4 \mathrm{~s}, 8 \mathrm{~s}$ and Dryden gust simulations showed reductions of $66 \%, 40 \%$, and $76 \%$, respectively. The figure also shows that this is accomplished with very little control effort, with the maximum aileron deflection of approximately $0.4^{\circ}$. This low control deflection is due to the fact that the curvature is not changing much, which is evident in the tip deflection plots. The maximum tip deflections is $0.015 \mathrm{~m}$.

Due to the fact that the lateral controller tracks roll commands using the ailerons, this controller did not perform well when the curvature reducing LQR commands were simply added to the aileron commands from the lateral controller. The presence of four moveable tails provides flexibility in the design of controllers. A new control scheme was developed using the two inboard elevators to control the aircraft pitch and the two outboard elevators to control roll, and has shown promising preliminary results. This approach leaves the ailerons free to control the curvature. 

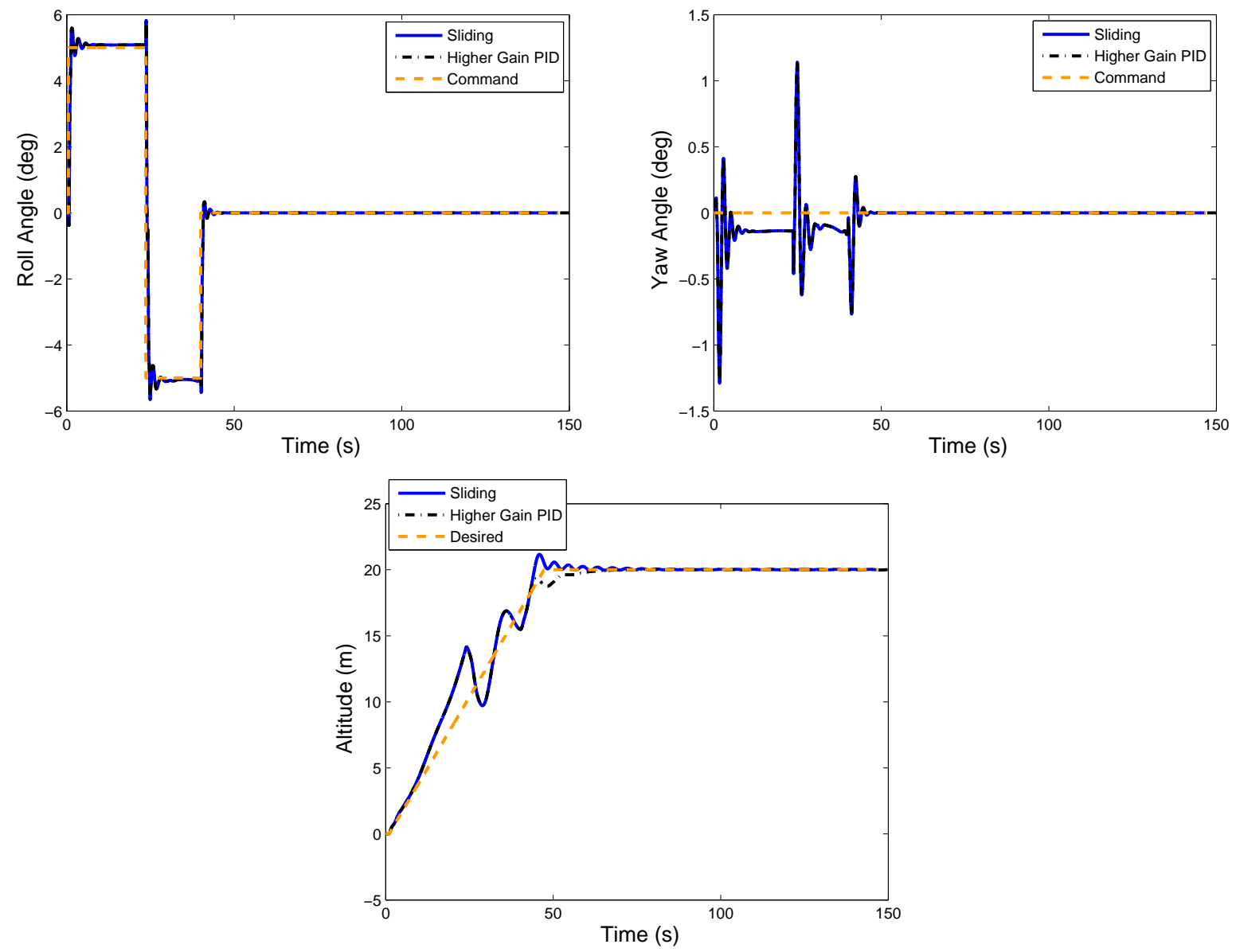

Figure 13. Response to simultaneous climb and roll commands. 

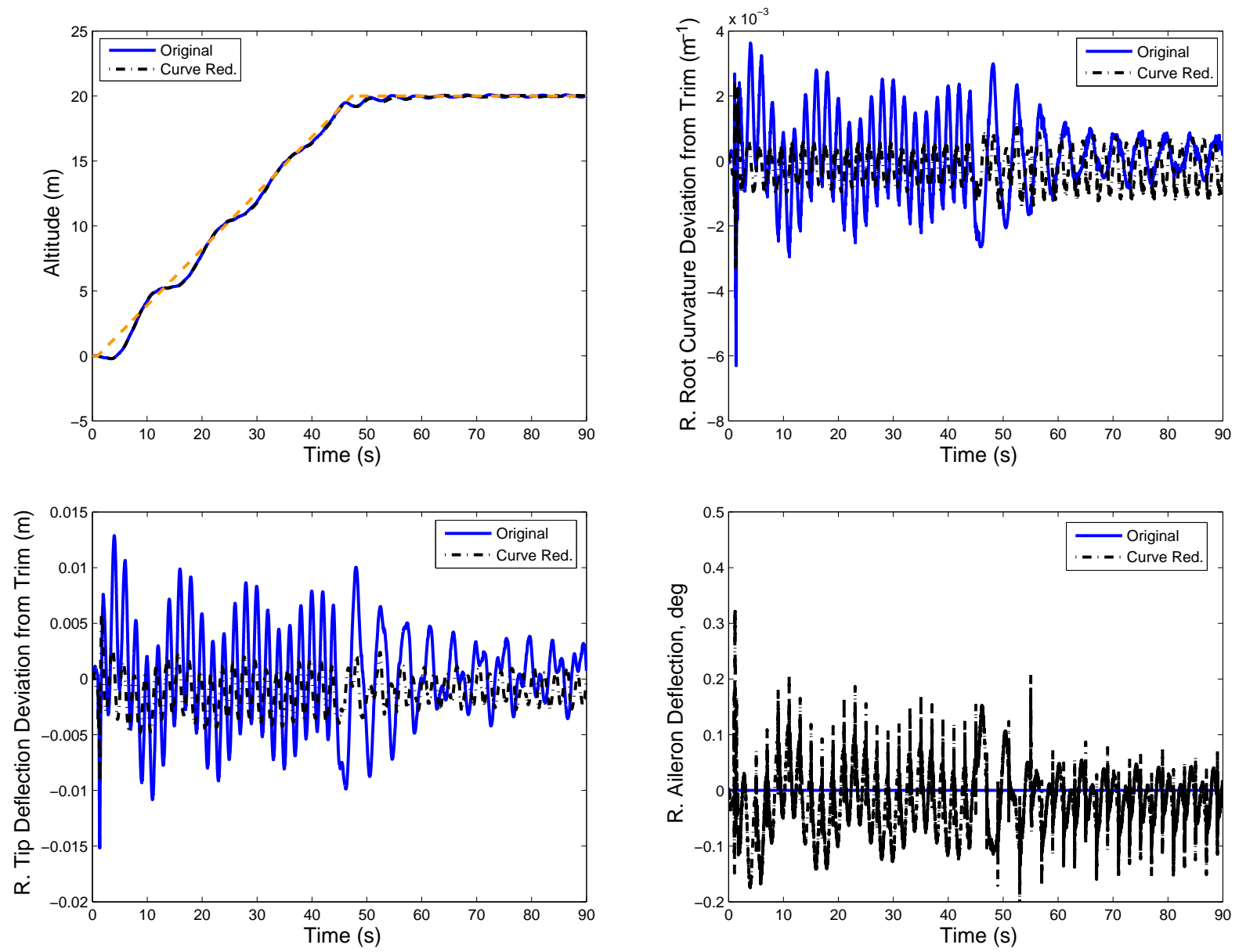

Figure 14. Comparison of altitude tracking with and without curvature reduction controller in $2 \mathrm{~s}$ duration gust. 

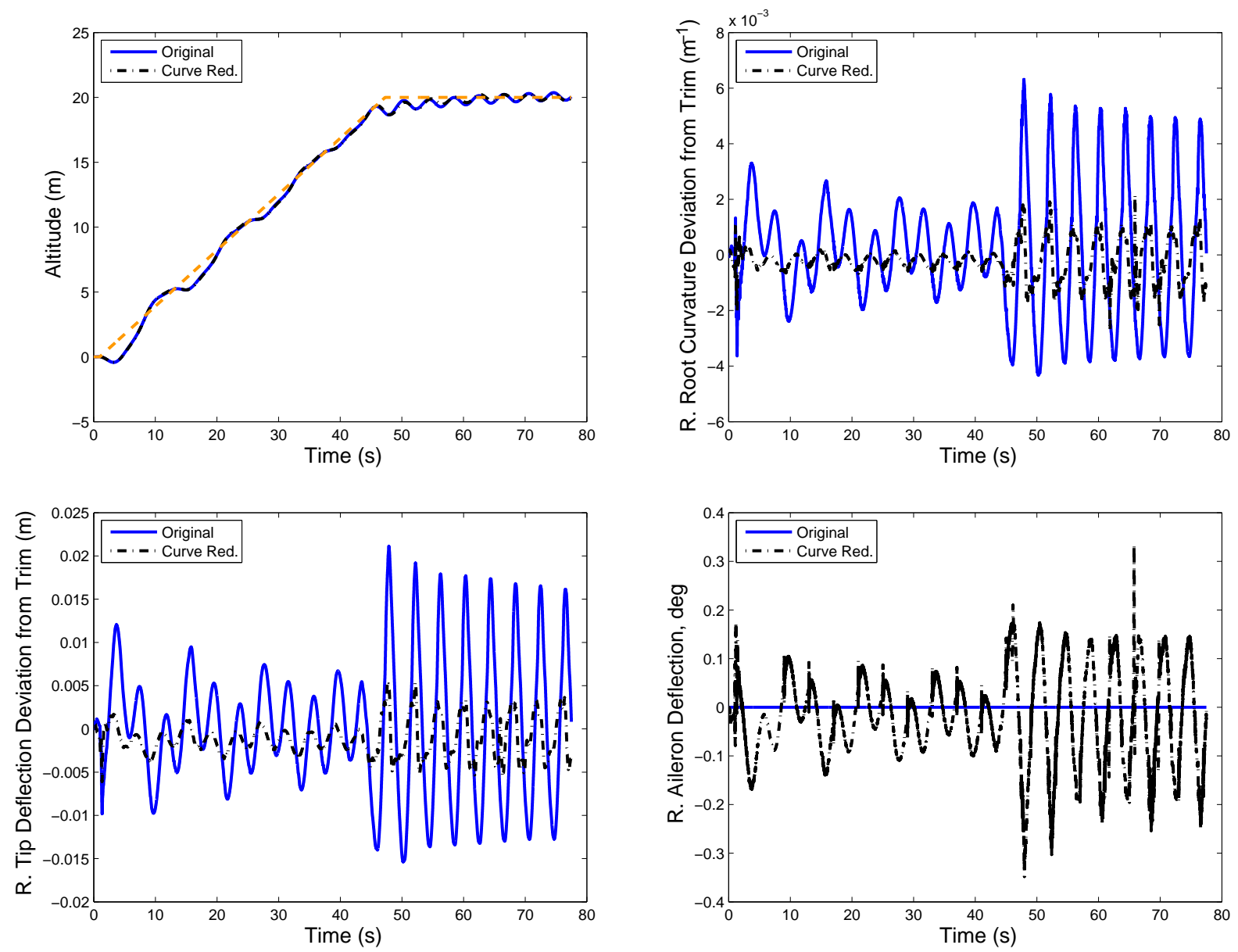

Figure 15. Comparison of altitude tracking with and without curvature reduction controller in $4 \mathrm{~s}$ duration gust. 

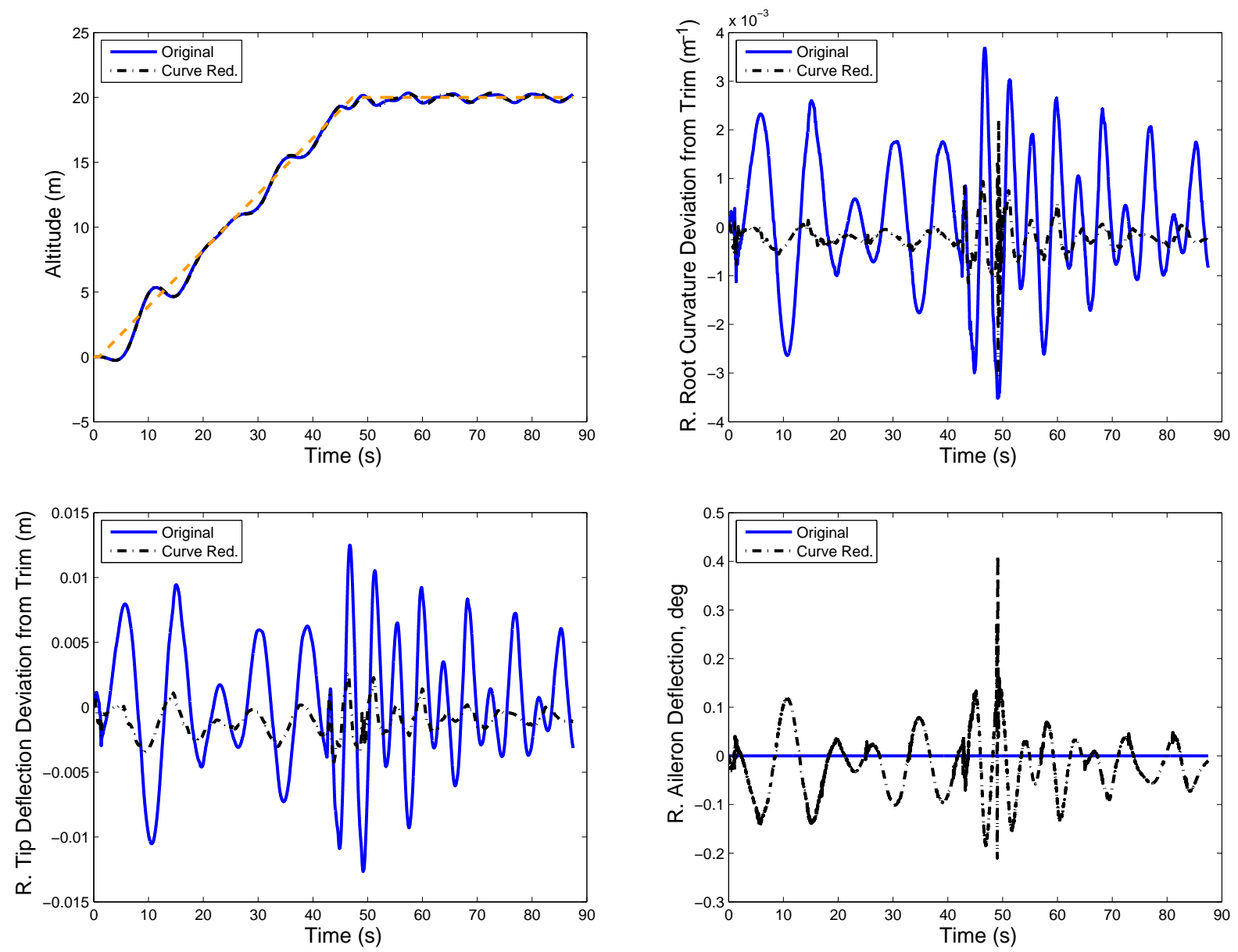

Figure 16. Comparison of altitude tracking with and without curvature reduction controller in $8 \mathrm{~s}$ duration gust. 

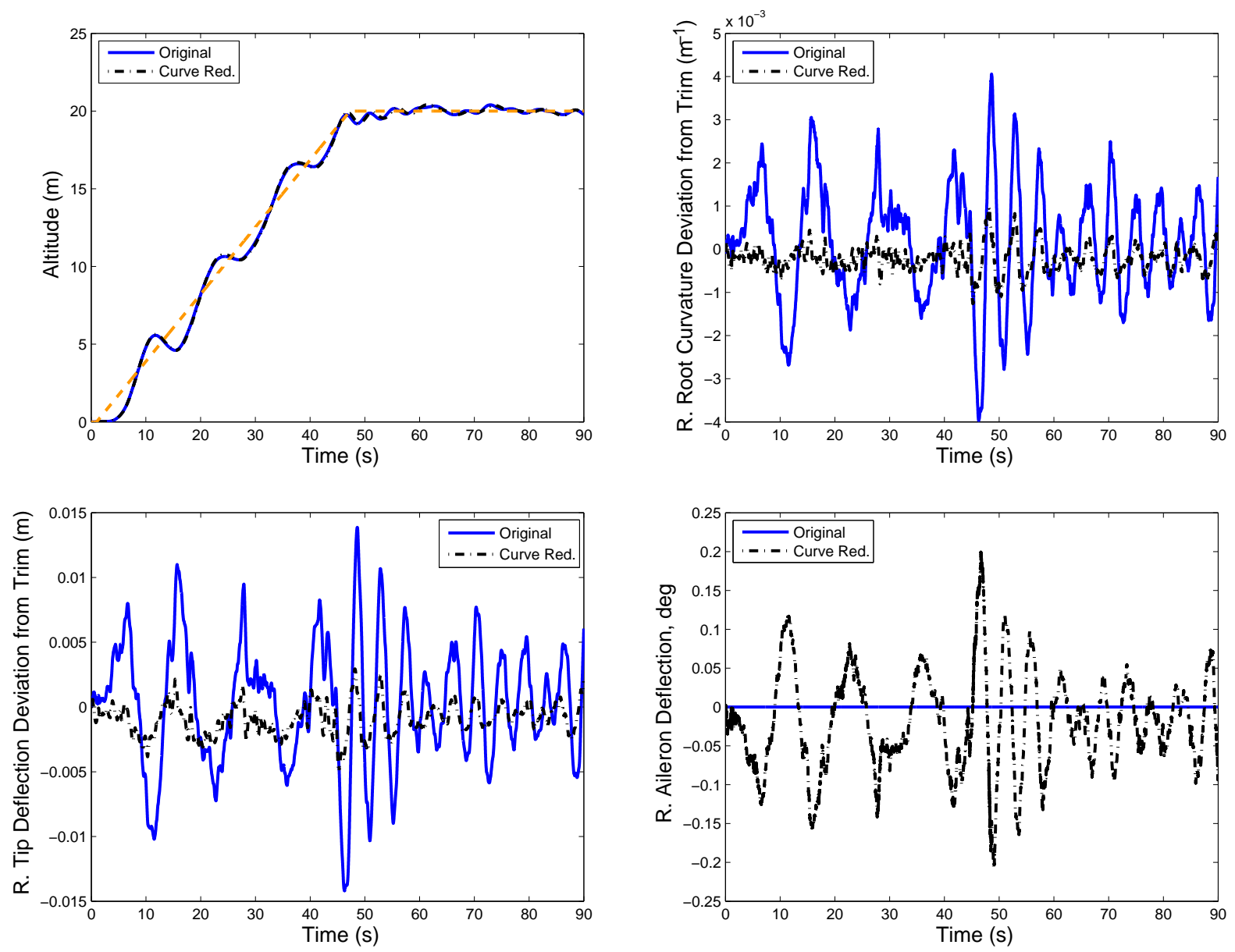

Figure 17. Comparison of altitude tracking with and without curvature reduction controller in Dryden gust. 


\section{Summary}

A method for controlling a VFA trajectory was presented. A two-loop control scheme consisting of a PID controlled outer loop and a dynamic inversion inner loop was demonstrated. Two modifications to the outer loop, one with higher PID gains in leveling off flight phase and another with a sliding mode controller, have been shown to yield good tracking of altitude change commands on the fully nonlinear aircraft model with wind gusts. A lateral controller based on an LQG design using a reduced order linearized aircraft model was presented and shown to track roll and yaw angle commands on the nonlinear aircraft model in presence of wind gusts. Finally, a reduction in root curvature, and therefore a reduction in bending moment, of up to $76 \%$ has been demonstrated by coordinated control of ailerons with an LQR controller reliant on full elastic state feedback during maneuvering flight in gust.

\section{References}

${ }^{1}$ Pail, M., Hodges, D., and Cesnik, C., "Nonlinear Aeroelasticity and Flight Dynamics of High-Altitude Long Endurance Aircraft," Jouranl of Aircraft, Vol. 38, No. 1, 2001, pp. 88-94.

${ }^{2}$ Tilman, C., Flick, P., and Martin, C., "High-Altitude Long Endurance Technologies for SensorCraft," RTO AVT Symposium on Novel Vehicle Concepts and Emerging Vehicle Technologies, Brussels, Belgium, April 2003, MP-104-P-26-1.

${ }^{3}$ Shearer, C. and Cesnik, C., "Trajectory Control for Very Flexible Aircraft," Journal of Guidance, Control, and Dynamics, Vol. 31, No. 2, 2008, pp. 340-357.

${ }^{4}$ Noll, T., Brown, J., Perez-Davis, M., Ishmael, S., Tiffany, G., and Gaier, M., "Investigation of the Helios Prototype Aircraft Mishap," Tech. rep., NASA, Jan. 2004.

${ }^{5} \mathrm{Su}$, W. and Cesnik, C., "Dynamic Response of Highly Flexible Flying Wings," AIAA Journal, Vol. 49, No. 6, 2001, pp. 324-339.

${ }^{6}$ Zhou, Z. and Ren, G., "Multibody Dynamic Approach of Flight Dynamics and Nonlinear Aeroelasticy of Flexible Aircraft," AIAA Journal, Vol. 49, No. 1, 2011.

${ }^{7}$ Peters, D. A. and Johnson, M., "Finite State Airloads for Deformable Airfoils on Fixed and Rotating Wings," Symposium on Aeroelasticity and Fluid/Structure Interaction/Proceedings of the Winter Annual Meeting, Fairfield, NJ, 1994.

${ }^{8}$ Shearer, C. and Cesnik, C., "Nonlinear Flight Dynamics of Very Flexible Aircraft," Journal of Aircraft, Vol. 44, No. 5, 2007, pp. 1528-1545.

${ }^{9}$ Cesnik, C. E. S., Senatore, P. J., Su, W., Atkins, E. M., and Shearer, C. M., "XHALE: A Very Flexible Unmanned Aerial Vehicle for Nonlinear Aeroelastic Tests," AIAA Journal, Vol. 50, No. 12, 2012

10 anon, "Federal Aviation Regulation (FAR) 23," Tech. rep., Federal Aviation Administration.

${ }^{11}$ anon, "Vulture II Broad Agency Announcement," Tech. rep., Defense Advanced Research Projects Agency.

${ }^{12}$ Dillsaver, M., Cesnik, C., and Kolmanovsky, I., "Gust response sensitivity of very flexible aircraft," AIAA Atmospheric Flight Mechanics Conference, Minneapolis, MN, August 2012.

${ }^{13}$ Hoblit, F. M., Gust Loads on Aircraft: Concepts and Applications, AIAA Education Series, 1988.

${ }^{14}$ Slotine, J. and Li, W., Applied Nonlinear Control, Prentice Hall, 1991.

${ }^{15}$ Lin, C. F., Advanced Control System Design, Prentice Hall, 1994.

${ }^{16}$ Stefani, R. T., Shahian, B., Savent, C. J., and Hostetter, G., Design of Feedback Control Systems, Oxford University Press, 2002.

${ }^{17}$ Khalil, H. K., Nonlinear Systems, Third Edition, Prentice Hall, 2002.

${ }^{18}$ Skogestag, S. and Postlethwaite, I., Multivariable Feedback Control Analysis and Design, John Wiley and Sons, Inc, 2005 .

\section{Appendix}

This section given an overview and theoretical background for the control topics used in the development of this paper.

\section{A. LQR/LQG Control Design}

The LQR is a popular control technique, see e.g. Ref. [9], that applies to a linear time-invariant system model of the form:

$$
\begin{aligned}
& \dot{x}=A x+B u, \\
& y=C x+D u,
\end{aligned}
$$

where $x \in \mathbb{R}^{n}$ is the state vector, $u \in \mathbb{R}^{m}$ is the control vector, and $y \in \mathbb{R}^{p}$ is the measured output vector. 
The LQR methodology provides a systematic way to choose a state feedback gain matrix $K$ by minimizing the cost function:

$$
J=\int_{0}^{\infty}\left(x^{T} Q x+u^{T} R u\right) d t
$$

where $Q$ is a positive semi-definite state weighting matrix and $R$ is a positive definite control weighting matrix. A matrix $P$ is found by solving the algebraic Riccati Equation,

$$
P A+A^{T} P+Q-P B R^{-1} B^{T} P=0,
$$

Then the optimal gain matrix is found using

$$
K=R^{-1} B^{T} P,
$$

and the LQR controller is then given by $u=-K x$. A Linear Quadratic Gaussian (LQG) controller combines an LQR controller that assumes full state measurement with a Kalman filter, used for state estimation.

The LQG design problem, applied to Eqns. (46) and (47), is to find the control matrix, $K$ that minimizes the cost function:

$$
J=\lim _{T \rightarrow \infty} \frac{1}{T} E\left\{\int_{0}^{T}\left(x^{T} Q x+u^{T} R u\right) d t\right\},
$$

with the control input $u$ is given by ${ }^{15}$

$$
u=-K \hat{x},
$$

where $\hat{x}$ is the state estimate determined by the Kalman filter.

$$
\dot{\hat{x}}=A \hat{x}+B u+L(y-C \hat{x}),
$$

where $L$ is the optimal observer gain calculated using

$$
L=\Sigma C^{T} R^{-1},
$$

$\Sigma$ is the positive semi-definite solution of the algebraic Riccatti equation,: ${ }^{16}$

$$
A \Sigma+\Sigma A^{T}+Q_{o}-\Sigma C^{T} R_{o}^{-1} C \Sigma=0,
$$

and $Q_{o}$ and $R_{o}$ are the intensity matrices of the process and sensor noise.

\section{B. Dynamic Inversion Control Design}

Nonlinear Dynamic Inversion (DI) is a control technique which uses nonlinear transformations and feedback to obtain a linear system model, to which standard linear control techniques can be applied. ${ }^{3,17}$ Consider a nonlinear system model in control affine form,

$$
\begin{gathered}
\dot{x}=f(x)+g(x) u, \\
y=h(x),
\end{gathered}
$$

where $x$ is the state vector, $u$ is the control vector, and $y$ is the output vector, which is assumed to be of the same dimension as the input vector. The derivative $\dot{y}$ is given by:

$$
\dot{y}=\frac{\partial h}{\partial x} f(x)+\frac{\partial h}{\partial x} g(x) u
$$

Assuming the matrix $\left[\frac{\partial h}{\partial x} g(x)\right]^{-1}$ is invertible, the control input $u$ can be calculated as:

$$
u=\left[\frac{\partial h}{\partial x} g(x)\right]^{-1}\left(v-\frac{\partial h}{\partial x} f(x)\right)
$$


leading to $\dot{y}=v$. The control signal, $u$, can be determined by

$$
v=-K y,
$$

where the gain matrix, $K$, is found using linear control techniques.

\section{Model Order Reduction}

Often when dealing with systems with a large number of states, it is desirable to reduce the order of the system, while replicating its behavior. One method for accomplishing this is through balanced truncation. Given system model in the form (46) and (47), the controllability and observability gramians, $P$ and $Q$ respectively, are given by:

$$
\begin{aligned}
& P=\int_{0}^{\infty} e^{A \tau} B B^{T} e^{A^{T} \tau} d \tau, \\
& Q=\int_{0}^{\infty} e^{A^{T} \tau} C^{T} C e^{A \tau} d \tau .
\end{aligned}
$$

The gramians can be found as solutions to the following Lyapunov equations, ${ }^{18}$

$$
\begin{aligned}
& A P+P A^{T}+B B^{T}=0 \\
& A^{T} Q+Q A+C^{T} C=0 .
\end{aligned}
$$

A balanced realization of the system is an asymptotically stable, minimal realization for which the gramians are equal, i.e. $P=Q$. The Hankel singular values, $\sigma_{i}$ of the system are defined as ${ }^{18}$

$$
\sigma_{i}=\sqrt{\lambda_{i}(P Q)}
$$

where $\lambda_{i}$ denotes the eigenvalues of the system. The Hankel singular values provide a measure of the relative contributions of each state to the input-output behavior of the entire system. The balanced truncation method simply removes the states with the lowest Hankel singular values, leaving a lower order system with similar input-output behavior to the original system. 